\title{
MicroRNA-298 inhibits malignant phenotypes of epithelial ovarian cancer by regulating the expression of EZH2
}

\author{
FENMEI ZHOU ${ }^{1 *}$, JUAN CHEN ${ }^{2 *}$ and HAIRONG WANG ${ }^{1}$ \\ ${ }^{1}$ Department of Obstetrics and Gynecology, Huai'an First People's Hospital, Nanjing Medical University, \\ Huai'an, Jiangsu 223300; ${ }^{2}$ Huai'an Maternal and Child Healthcare Center, Huai'an, Jiangsu 223302, P.R. China
}

Received May 22, 2015; Accepted September 1, 2016

DOI: $10.3892 / \mathrm{ol} .2016 .5204$

\begin{abstract}
MicroRNA (miRNA or miR)-298 has been reported to be downregulated and to modify the expression of the polycomb protein enhancer of zeste $2(\mathrm{EZH} 2)$ in recurrent epithelial ovarian cancer (EOC). To date, no functional evidence of a miR-298-EZH2 axis in EOC has been documented. The present study aimed to investigate the associations of miR-298 and/or EZH2 expression with clinicopathological features of EOC patients, and revealed their roles in cell motility based on EOC cell lines. Reverse transcription-quantitative polymerase chain reaction was performed to detect the expression levels of miR-298 and EZH2 messenger RNA in human EOC tissues and cell lines. Wound healing and transwell assays were performed to determine the function of the miR-298-EZH2 axis on cell migration and invasion, respectively. Compared with normal tissues, miR-298 expression was significantly downregulated, while EZH2 expression was significantly upregulated, in human EOC tissues (both $\mathrm{P}=0.001$ ). In addition, miR-298 downregulation and EZH2 upregulation were significantly associated with high clinical stage (both $\mathrm{P}=0.01$ ) and pathological grade (both $\mathrm{P}=0.02$ ) of EOC patients. Furthermore, the ectopic expression of miR-298 could efficiently inhibit cell migration and invasion. Notably, the overexpression of EZH2 could restore the cell migration and invasion abilities suppressed by miR-298. Our data offer convincing evidence that the dysregulation of the miR-298-EZH2 axis may be important in tumor progression of EOC patients. The present study also confirmed a tumor-suppressive role of miR-298 in modulating EOC cell motility by regulating the expression of EZH2, implying its potential as a novel miRNA-based therapeutic target for the treatment of human EOC.
\end{abstract}

Correspondence to: Professor Hairong Wang, Department of Obstetrics and Gynecology, Huai'an First People's Hospital, Nanjing Medical University, 6 Beijing Road West, Huai'an, Jiangsu 223300, P.R. China

E-mail:whrjsha@163.com

*Contributed equally

Key words: epithelial ovarian cancer, microRNA-298, enhancer of zeste 2 , clinicopathological feature, migration, invasion

\section{Introduction}

As the leading cause of mortality due to gynecological malignancies in women worldwide, epithelial ovarian cancer (EOC) originates from the ovarian surface, inclusion cysts in the ovarian parenchyma or the nearby distal fallopian tube epithelium, and is characterized by a response to cytotoxic chemotherapy at an early stage, followed by recurrence and disease progression frequently $(1,2)$. According to the reports of the National Cancer Institute (Rockville, MD, USA), EOC caused an estimated 15,500 mortalities in the USA in 2012 (3). In China, it has also been a major problem over the past few decades, with high mortality (4). Since $>70 \%$ of EOC patients are diagnosed in advanced stages, with distant metastases at the time of diagnosis, the overall five-year survival rate is $20-30 \%(5,6)$. Given this scenario, it is of great clinical significance to develop novel and more efficient therapeutic strategies to combat EOC.

MicroRNAs (miRNAs or miRs) represent a diverse class of evolutionarily conserved, small (21-23 nucleotides in length), non-protein-coding RNAs (7). Functionally, miRNAs execute post-transcriptional regulation by binding to the 3 -untranslated region of their target genes (8). Recent evidence has shown that each miRNA can regulate multiple target genes, leading to the potential influence of miRNAs on various cellular activities (9). Pathologically, half of the human miRNAs have been identified to be located in cancer-associated genomic regions, and can act as either tumor suppressors or oncogenes according to the functions of their target genes (10). Growing evidence suggests that the aberrant expression of miRNAs may be involved in the pathogenesis of numerous cancers (11). Particularly in EOC, there have been a number of studies that have reported the differentially regulated miRNAs in cancer cells compared with normal controls, and have confirmed their critical role in cancer progression (12-14). For instance, Wang et al (12) revealed that miR-182 was upregulated in ovarian cancer tissues and cell lines, and could function as an oncogene to promote cancer cell growth, invasion and chemoresistance by targeting programmed cell death 4 . Zhang et al (13) reported that miR-124 was downregulated in ovarian cancer specimens as well as in cell lines, and suppressed cancer cell migration and invasion by targeting sphingosine kinase 1 . Wu et al (14) demonstrated that miR-145 was downregulated in human ovarian cancer, and inhibited cancer cell growth and invasion 
Table I. Association of miR-298 downregulation and/or polycomb protein EZH2 upregulation with clinicopathological features of epithelial ovarian cancer tissues.

\begin{tabular}{|c|c|c|c|c|c|c|c|}
\hline Features & $\begin{array}{c}\text { No. } \\
\text { of patients }\end{array}$ & miR-298-low & P-value & EZH2-high & P-value & $\begin{array}{l}\text { miR-298-low/ } \\
\text { EZH2-high }\end{array}$ & P-value \\
\hline \multicolumn{8}{|l|}{ Age } \\
\hline$<55$ years & 45 & $23(51.11)$ & \multirow[t]{2}{*}{0.380} & $20(44.44)$ & \multirow[t]{2}{*}{0.260} & $18(40.00)$ & \multirow[t]{2}{*}{0.500} \\
\hline$\geq 55$ years & 55 & $30(54.55)$ & & $31(56.36)$ & & $22(40.00)$ & \\
\hline \multicolumn{8}{|c|}{ Histological type } \\
\hline Serous & 28 & $13(46.43)$ & \multirow[t]{2}{*}{0.290} & 15 (53.57) & \multirow[t]{2}{*}{0.180} & $8(28.57)$ & \multirow[t]{2}{*}{0.230} \\
\hline Non-serous & 82 & $40(48.78)$ & & $36(43.90)$ & & $32(39.02)$ & \\
\hline \multicolumn{8}{|c|}{ Residual tumor upon surgery } \\
\hline$<1 \mathrm{~cm}$ & 35 & $16(45.71)$ & \multirow[t]{2}{*}{0.210} & $15(42.86)$ & \multirow[t]{2}{*}{0.160} & $15(42.86)$ & \multirow[t]{2}{*}{0.320} \\
\hline$\geq 1 \mathrm{~cm}$ & 65 & 37 (56.92) & & $36(55.38)$ & & 25 (38.46) & \\
\hline \multicolumn{8}{|l|}{ Clinical stage } \\
\hline I-II & 80 & $35(43.75)$ & \multirow[t]{2}{*}{0.010} & $33(41.25)$ & \multirow[t]{2}{*}{0.010} & $24(30.00)$ & \multirow[t]{2}{*}{0.008} \\
\hline III-IV & 20 & $18(90.00)$ & & $17(85.00)$ & & $16(80.00)$ & \\
\hline \multicolumn{8}{|c|}{ Pathological grade } \\
\hline $1-2$ & 70 & $28(40.00)$ & \multirow[t]{2}{*}{0.020} & $26(37.14)$ & \multirow[t]{2}{*}{0.020} & $15(21.43)$ & \multirow[t]{2}{*}{0.010} \\
\hline 3 & 30 & $25(83.33)$ & & $25(83.33)$ & & $25(83.33)$ & \\
\hline
\end{tabular}

miR, microRNA; EZH2, enhancer of zeste 2.

by targeting p70S6 kinase 1 and mucin 1 . Collectively, these studies suggest the involvement of miRNAs in the carcinogenesis of EOC. A recent study has shown that miR-298 was downregulated and could regulate the expression of the polycomb protein enhancer of zeste $2(\mathrm{EZH} 2)$ in recurrent EOC (15). To date, no functional evidence of a miR-298-EZH2 axis in EOC has been documented.

In the current study, the potential involvement of the miR-298-EZH2 axis in EOC was investigated. The expression levels of miR-298 and EZH2 messenger (m) RNA in human EOC tissues were detected, and the associations of miR-298 and/or EZH2 expression with clinicopathological features of EOC patients were analyzed. In addition, a potential role of the miR-298-EZH2 axis on cell motility was also investigated on EOC cell lines. The present study provides an improved understanding of the molecular mechanisms responsible for the aggressive nature of human EOC.

\section{Materials and methods}

Patients and ethics. In total, 100 EOC tissue specimens and 20 normal ovarian tissue specimens were collected from surgery during January 2008 to December 2012, snap-frozen in liquid nitrogen and then stored at $-80^{\circ} \mathrm{C}$, at the Department of Obstetrics and Gynecology, Huai'an First People's Hospital, Nanjing Medical University (Huai'an, China). Written informed consent was obtained from all patients, and the study was approved by the Ethics Committee of Huai'an First People's Hospital, Nanjing Medical University.

None of the EOC patients were treated with radiotherapy, chemotherapy or hormonal therapy prior to surgery. Surgical staging was established based on the International Federation of Gynecology and Obstetrics system (16). The clinicopathological features of the 100 EOC patients were summarized in Table I. All 20 normal ovarian tissues were obtained from women who underwent hysterectomies for benign disease.

Cell culture. Two EOC cell lines, SKOV3 and OVCAR3, were obtained from the Shanghai Institute of Cell Biology, China Academy of Sciences (Shanghai, China), and were cultured in RPMI-1640 medium (Gibco; Thermo Fisher Scientific, Inc., Waltham, MA, USA) supplemented with $10 \%$ fetal calf serum (Gibco; Thermo Fisher Scientific, Inc.) in a humidified atmosphere of $5 \% \mathrm{CO}_{2}$ at $37^{\circ} \mathrm{C}$.

RNA and miRNA extraction. Total RNAs were extracted from tissues and cells with TRIzol reagent (Invitrogen; Thermo Fisher Scientific, Inc.) according to the manufacturer's protocol. In addition, total miRNA was extracted from tissues and cells using the mirVana miRNA Isolation kit (Ambion; Thermo Fisher Scientific, Inc.) according to the manufacturer's protocol.

Reverse transcription-quantitative polymerase chain reaction (RT-qPCR) assay. RT-qPCR assay was performed to detect the expression levels of miR-298 and EZH2 mRNA in EOC tissues and cells. In brief, $10 \mu \mathrm{g}$ of small nuclear RNA and $20 \mu \mathrm{g}$ of total RNA were subjected to RT. Single-stranded complementary (c) DNA was synthesized using the PrimeScript RT Reagent kit (Promega Corporation, Madison, WI, USA). Subsequently, the single-stranded cDNA was used for the amplification of mature miR-298, EZH2 and the endogenous controls [U6 small nuclear RNA B and glyceraldehyde 3-phosphate dehydrogenase (GAPDH)] by PCR. The PCR primers used were as follows: miR-298 forward, 5'-ACA CTC AGC TGG GAG CAG AAG CAG GGA G-3' and reverse, 5'-GGT GTC GTG GAG TCG-3'; U6 forward, 5'-CGC TTC 


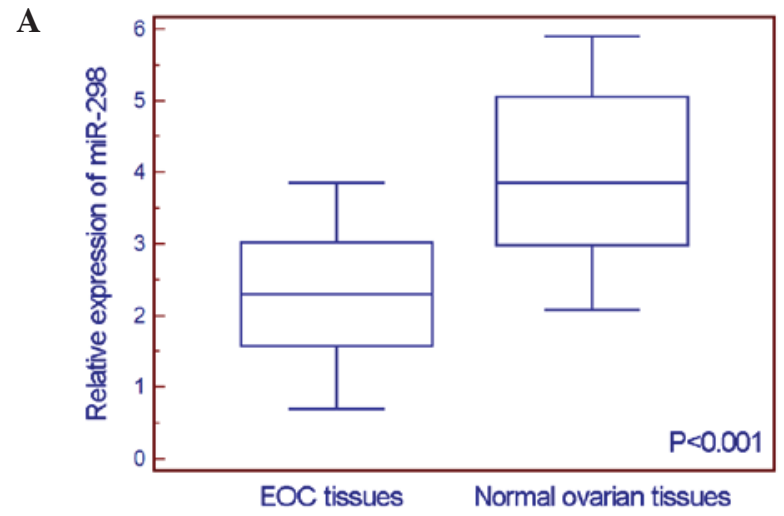

B

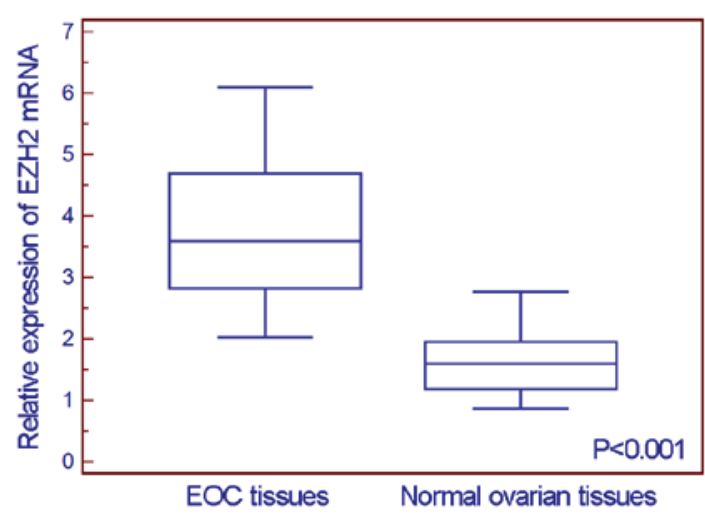

C

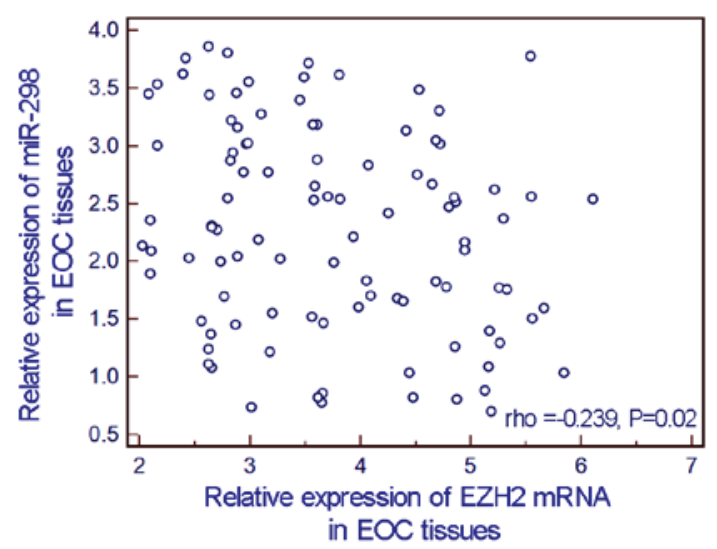

Figure 1. Expression levels of miR-298 and EZH2 mRNA in 100 EOC tissue specimens and 20 normal ovarian tissue specimens detected by reverse transcription-quantitative polymerase chain reaction assay and normalized to the levels of U6 small nuclear RNA B. (A) The expression level of miR-298 in EOC tissues was significantly lower than that in normal ovarian tissues (EOC vs. normal: $2.29 \pm 0.88$ vs. $4.02 \pm 1.19, \mathrm{P}<0.001$ ). (B) The expression level of EZH2 mRNA in EOC tissues was significantly higher than that in normal ovarian tissues (EOC vs. normal: $3.73 \pm 1.07$ vs. $1.65 \pm 0.58, \mathrm{P}<0.001$ ). (C) An inverse correlation exists between miR-298 and EZH2 mRNA expression levels (Spearman's correlation coefficient $=-0.239, \mathrm{P}=0.02$ ). $\mathrm{miR}$, microRNA; EZH2, enhancer of zeste 2; mRNA, messenger RNA; EOC, epithelial ovarian cancer.

GGC AGC ACA TAT AC-3' and reverse, 5'-CAG GGG CCA TGC TAA TCT T-3'; EZH2 forward, 5'-GGG AGA CTA TTC TTG ATG GGA AG-3' and reverse, 5'-ACT GCA ACG TAG GTC CCT GA-3'; and GAPDH forward, 5'-GGC GGC ACC ACC ATG TAC CCT-3' and reverse, 5'-AGG GGC CGG ACT CGT CAT ACT-3'. The PCR conditions were: Initial denaturation at $95^{\circ} \mathrm{C}$ for $3 \mathrm{~min}$, followed by 40 cycles of $95^{\circ} \mathrm{C}$ for $15 \mathrm{sec}, 62^{\circ} \mathrm{C}$ for $30 \mathrm{sec}$ and $72^{\circ} \mathrm{C}$ for $30 \mathrm{sec}$.

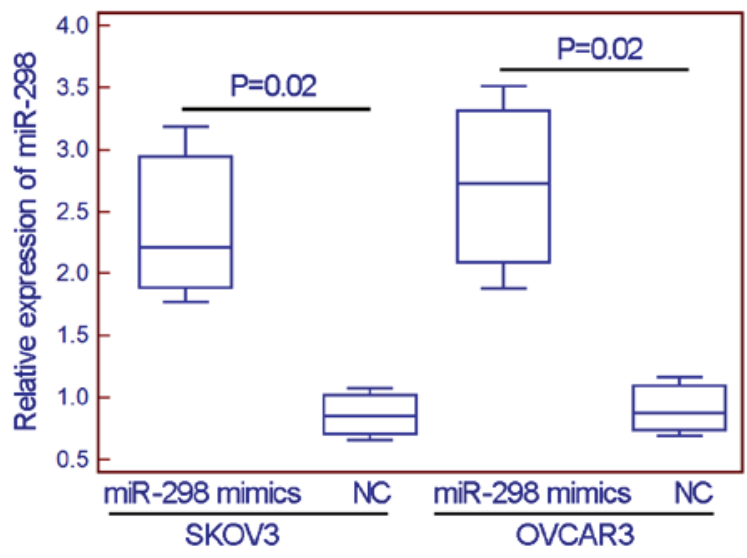

Figure 2. Overexpression of miR-298 in SKOV3 and OVCAR3 cells transfected with miR-298 mimics and NC mimics. miR, microRNA; NC, negative control.

qPCR was performed using SYBR Green PCR Master Mix (Applied Biosystems; Thermo Fisher Scientific, Inc.) on an ABI 7300HT Real-Time PCR system (Applied Biosystems; Thermo Fisher Scientific, Inc.). Standard curves were generated, and the relative amount of miR-298 or EZH2 was normalized to the amount of U6 or GAPDH, respectively. Gene expression was normalized to endogenous controls, and fold-changes were calculated using the $2^{-\Delta \Delta C q} \operatorname{method}(17)$.

Plasmid construct and transfection. miR-298 mimics and mimic controls were purchased from Shanghai GenePharma Co., Ltd. (Shanghai, China). According to the previous report of Zhang et al (18), the full-length EZH2 cDNA was obtained by PCR using an expressed sequence tag clone as template, and constructed into the pCEP4 expression vector to express EZH2. The cells were transfected using Lipofectamine 2000 (Invitrogen; Thermo Fisher Scientific, Inc.), according to the manufacturer's transfection protocol.

Western blot analysis. Upon $72 \mathrm{~h}$ of transient transfection, EOC cells were harvested and lysed using radioimmunoprecipitation assay buffer $[50 \mathrm{mM}$ Tris- $\mathrm{HCl}$ (pH 8.8), $298 \mathrm{mM} \mathrm{NaCl}, 1 \%$ NP- $40,1 \%$ sodium deoxycholate and $0.1 \%$ sodium dodecyl sulfate (SDS)]. Then, western blot analysis was performed to detect the expression levels of EZH2 protein. The proteins were resolved on a $10 \%$ SDS denaturing polyacrylamide gel and transferred onto a nitrocellulose membrane. The membranes were blocked with 5\% nonfat milk in Tris-buffered saline with Tween-20 for $1 \mathrm{~h}$ at room temperature, and next incubated with anti-EZH2 antibody (dilution 1:250; sc-25383; Santa Cruz Biotechnology, Inc., Dallas, TX, USA) or anti-GAPDH antibody (dilution 1:250; sc-25778; Santa Cruz Biotechnology, Inc.) overnight at room temperature. Horseradish peroxidase-conjugated anti-rabbit secondary antibody (dilution 1:1,000; sc-2030; Santa Cruz Biotechnology, Inc.) was used for detection following incubation for $2 \mathrm{~h}$ at room temperature. GAPDH was used as an internal control for the normalization of candidate proteins. Protein expression was assessed by enhanced chemiluminescence with SuperSignal West Pico Chemiluminescent Substrate (Thermo Fisher Scientific, Inc.), followed by exposure to a chemiluminescent film (Pierce Biotechnology, Inc., Rockford, IL, USA). 
A
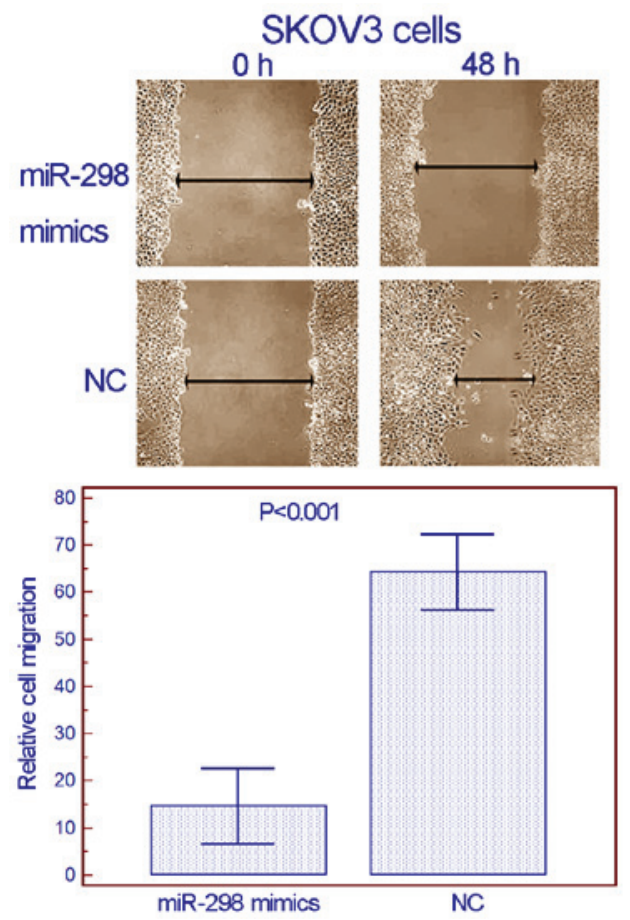

B
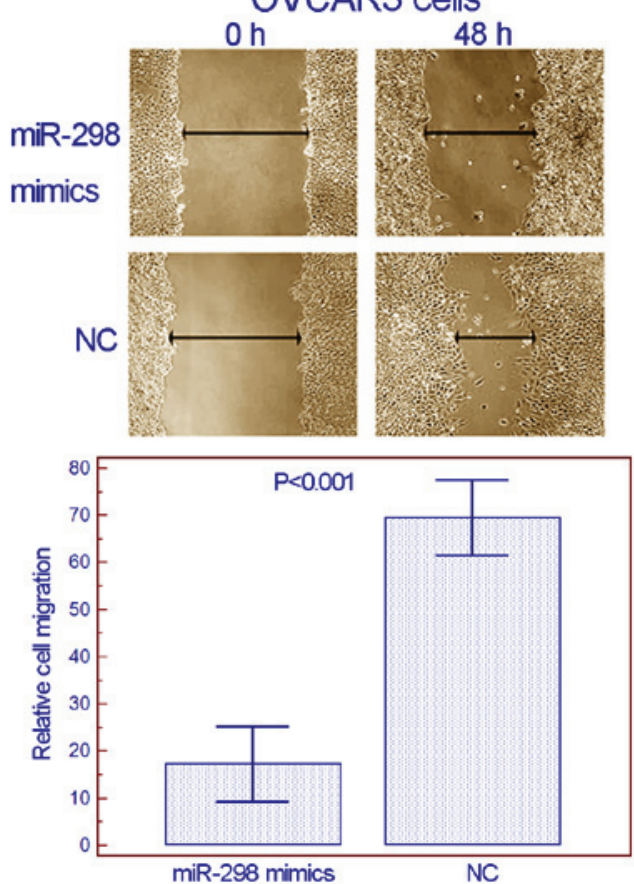

Figure 3. miR-298 inhibits the migration of (A) SKOV3 and (B) OVCAR3 cells transfected with miR-298 mimics and NC mimics. Magnification, x100. miR, microRNA; NC, negative control.

A
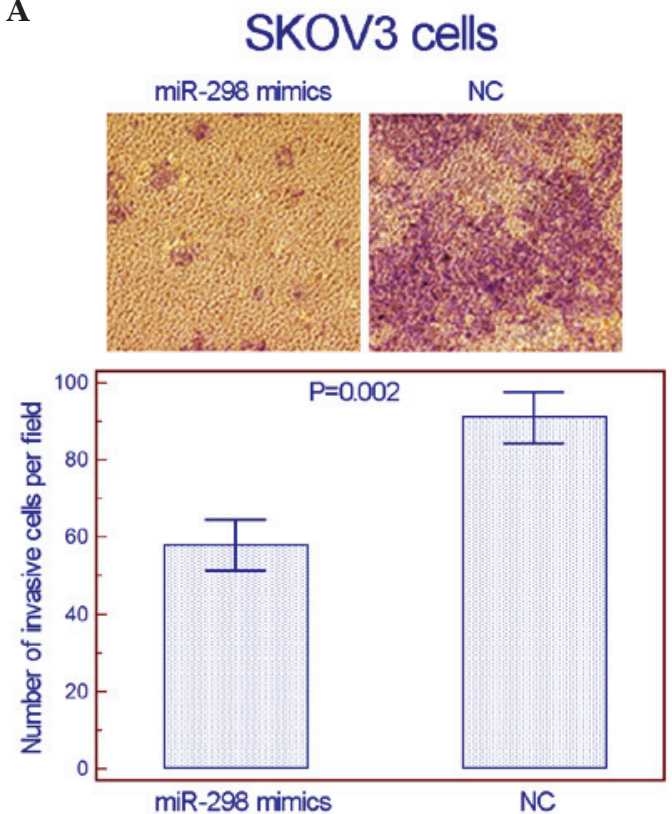

B

OVCAR3 cells
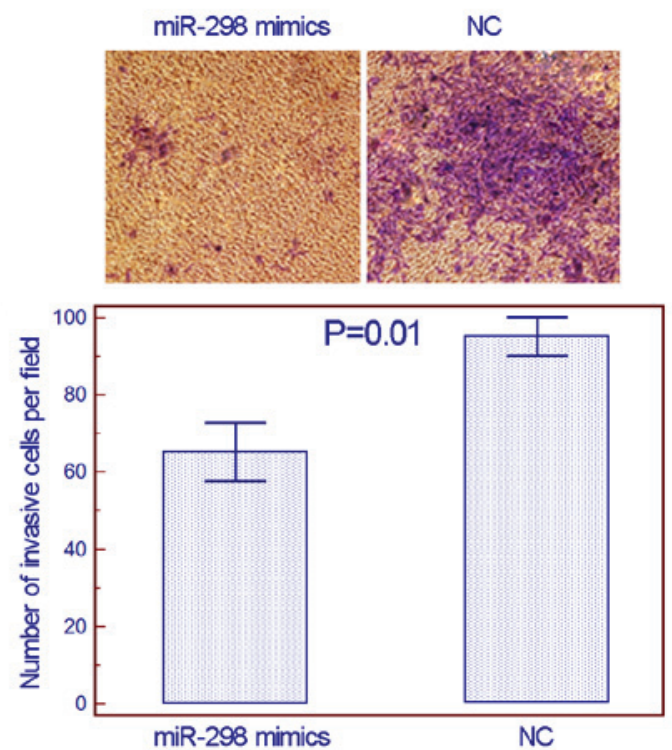

Figure 4. miR-298 inhibits the invasion of (A) SKOV3 and (B) OVCAR3 cells transfected with miR-298 mimics and NC mimics. Magnification, x100. miR, microRNA; NC, negative control.

In vitro migration assay. Wound healing assay was performed to evaluate the cell migration abilities of EOC cells transfected with miR-298 mimics/mimic controls or EZH2 expression vector/negative control (NC) vector, according to previous studies $(19,20)$. Briefly, $48 \mathrm{~h}$ after transfection, EOC cells were scraped with a $200-\mu 1$ pipette tip to create an artificial wound, and incubated with fresh medium containing mitomycin $\mathrm{C}$ (5 $\mu \mathrm{g} / \mathrm{ml}$; Applied Biosystems; Thermo Fisher Scientific, Inc.) for $12 \mathrm{~h}$. The fraction of cell coverage across the line was measured as the migration rate. All experiments were performed in triplicate.

In vitro invasion assay. Transwell assay was performed to evaluate the cell invasion ability of EOC cells transfected with miR-298 mimics/mimic controls or EZH2 expression vector/NC vector using Matrigel-coated cell culture chambers (8- $\mu \mathrm{m}$ pore size; EMD Millipore, Billerica, MA, USA). Briefly, $48 \mathrm{~h}$ after transfection, confluent EOC cells were resuspended 
A

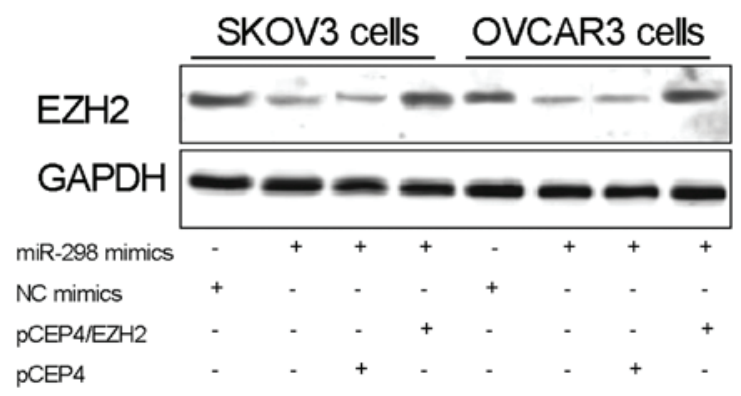

B

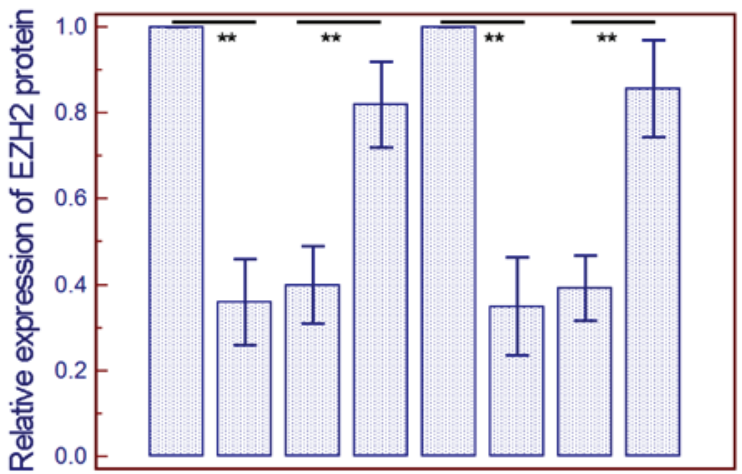

$\begin{array}{lllllllll}\text { miR-298 mimics } & - & + & + & + & - & + & + & + \\ \text { NC mimics } & + & - & - & - & + & - & - & - \\ \text { pCEP4/EZH2 } & - & - & - & + & - & - & - & + \\ \text { pCEP4 } & - & - & + & - & - & - & + & -\end{array}$

Figure 5. Expression levels of EZH2 protein, as detected by western blotting. (A) Western blot bands for the expression of EZH2 relative to that of GAPDH in the different groups. (B) The data confirmed that EZH2 expression was significantly upregulated, and could rescue the inhibition of EZH2 protein levels caused by miR-298 mimics. ${ }^{* *} \mathrm{P}<0.01$ vs. control. miR, microRNA; EZH2, enhancer of zeste 2; NC, negative control; GAPDH, glyceraldehyde 3-phosphate dehydrogenase.

in $200 \mu$ l serum-free RPMI 1640 medium and placed into the upper chamber of the insert with Matrigel. Medium with 5\% fetal bovine serum (Gibco; Thermo Fisher Scientific, Inc.) was added into the lower chamber as a chemoattractant. After $24 \mathrm{~h}$ of incubation, the cells remaining on the upper membrane were carefully removed. Cells that had invaded through the membrane were manually counted at x200 magnification from 10 different fields of each filter. All experiments were conducted in triplicate.

Statistical analysis. Statistical analyses were performed using SPSS version 19.0 software (IBM SPSS, Armonk, NY, USA). Data were expressed as the mean \pm standard deviation of $\geq 3$ independent experiments. Group differences were compared using one-way analysis of variance or two-tailed Student's $t$ test. The association between miR-298 and EZH2 mRNA expression was evaluated by Spearman's correlation. Comparisons between groups were performed using the $\chi^{2}$ test for categorical variables. $\mathrm{P}<0.05$ was considered to indicate a statistically significant difference.

\section{Results}

miR-298 is downregulated and EZH2 mRNA is upregulated in human EOC tissues. The comparisons of miR-298 and EZH2 expression detected by RT-qPCR assay between 100 EOC tissue specimens and 20 normal ovarian tissue specimens indicated that the expression level of miR-298 in EOC tissues was significantly lower than that in normal ovarian tissues (EOC vs. normal: $2.29 \pm 0.88$ vs. $4.02 \pm 1.19, \mathrm{P}<0.001$, Fig. $1 \mathrm{~A}$ ), while EZH2 mRNA expression was significantly upregulated in EOC tissues (EOC vs. normal: $3.73 \pm 1.07$ vs. $1.65 \pm 0.58$, $\mathrm{P}<0.001$, Fig. 1B). In addition, an inverse correlation existed between miR-298 and EZH2 mRNA expression levels (Spearman's correlation coefficient $=-0.239, P=0.020$, Fig. 1C).

miR-298 downregulation and EZH2 upregulation are associated with aggressive tumor progression of human EOC. To assess the associations of miR-298 and/or EZH2 mRNA expression with the clinicopathological features of human EOC, the 100 patients were divided into miR-298-low ( $\mathrm{n}=53$ ), miR-298-high $(n=47)$, EZH2-low $(n=49)$ and EZH2-high $(n=51)$ groups using the median values of miR-298 (2.31) or EZH2 (3.61) expression in EOC tissues, respectively. Of the 100 EOC patients, $13(13.00 \%), 40(40.00 \%), 36(36.00 \%)$, and $11(11.00 \%)$ patients belonged to the miR-298-low/EZH2-low, miR-298-low/EZH2-high, miR-298-high/EZH2-low and miR-298-high/EZH2-high groups, respectively.

As shown in Table I, miR-298 downregulation and EZH2 upregulation were significantly associated with high clinical stage (both $\mathrm{P}=0.01$ ) and pathological grade (both $\mathrm{P}=0.02$ ) of EOC patients. The EOC tissues with advanced clinical stage (III-IV) more frequently exhibited low miR-298 expression $(\mathrm{P}=0.010$, Table I) or high EZH2 expression $(\mathrm{P}=0.010$, Table I $)$ 
$\mathbf{A}$
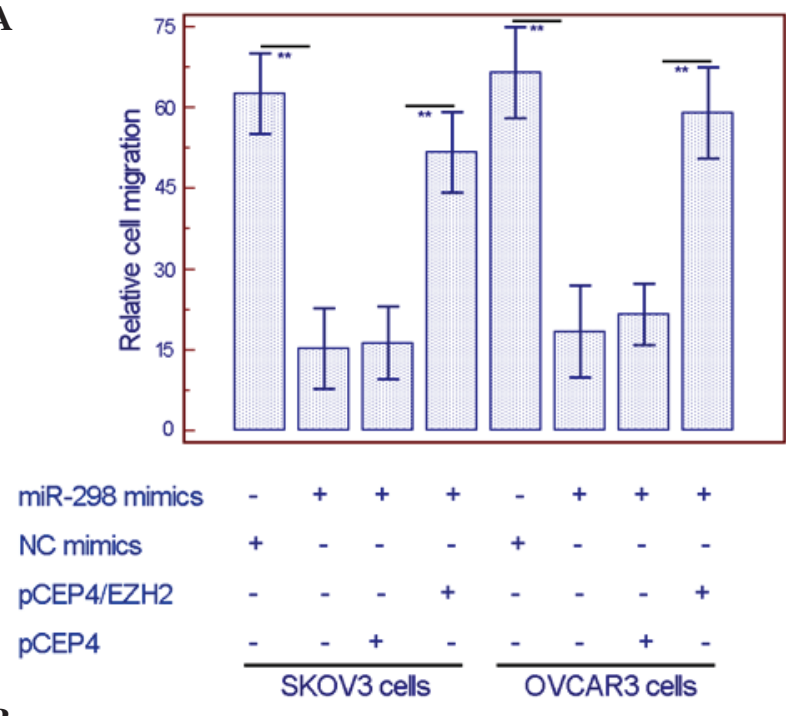

B
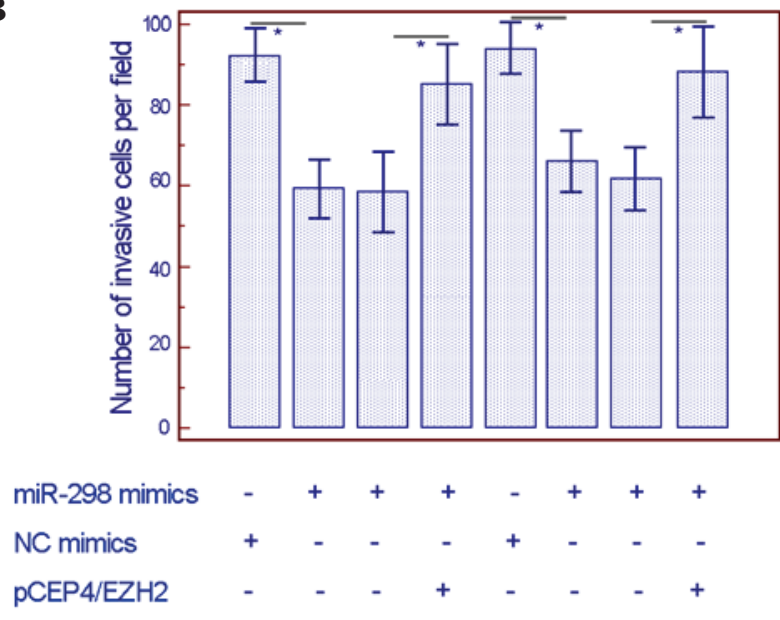

pCEP4

$$
\frac{-++-}{\text { SKOV3 cells }} \frac{-\quad+-}{\text { OVCAR3 cells }}
$$

Figure 6. (A) Wound healing assay and (B) transwell invasion assay demonstrated respectively that the overexpression of EZH2 could efficiently alleviate the miR-298 mimics-induced inhibition of the migration and invasion activities of SKOV3 and OVCAR3 cells. ${ }^{*} \mathrm{P}<0.05,{ }^{* *} \mathrm{P}<0.01$ vs. control. miR, microRNA; EZH2, enhancer of zeste 2; NC, negative control.

than those with low clinical stage (I-II). Additionally, miR-298 expression exhibited a trend that correlated with pathological grade $(\mathrm{P}=0.020$, Table $\mathrm{I})$. It was also observed that $\mathrm{EZH} 2$ was aberrantly upregulated in tumor tissues with high pathological grade compared with those with low pathological grade $(\mathrm{P}=0.020$, Table I). Of note, the associations of combined miR-298-low/EZH2-high expression with high clinical stage $(\mathrm{P}=0.008$, Table $\mathrm{I})$ and high pathological grade $(\mathrm{P}=0.010$, Table I) of EOC patients were both more remarkable than those of miR-298-low or EZH2-high alone.

miR-298 inhibits cell migration and invasion of EOC cells in vitro. To evaluate the effect of miR-298 on malignant phenotypes in EOC cells, miR-298 mimics were transfected into two EOC cell lines (SKOV3 and OVCAR3) to overexpress this miRNA (Fig. 2). From the wound healing assay, it was observed that enforced expression of miR-298 inhibited the migration activity of both SKOV3 and OVCAR3 cells (Fig. 3). Notably, in the transwell invasion assay, using Matrigel to simulate the extracellular matrix, the invasion activities of the two cell lines in vitro were suppressed following the transfection of miR-298 mimics (Fig. 4).

Overexpression of EZH2 can rescue the inhibitory effect induced by miR-298. To further validate whether EZH2 could mediate the tumor-suppressive effects of miR-298 on EOC cells, pCEP4/EZH2 was constructed to overexpress this gene. The data shown in Fig. 5A and B confirmed that EZH2 expression was significantly upregulated and could rescue the inhibition of the EZH2 protein levels caused by miR-298 mimics. Notably, the wound healing assay and the transwell invasion assay respectively demonstrated that the overexpression of EZH2 could efficiently alleviate the miR-298 mimics-induced inhibition of migration and invasion in both SKOV3 and OVCAR3 cells (Fig. 6).

\section{Discussion}

Increasing evidence has revealed that miRNAs function as important players and therapeutic targets in various human cancers, including EOC (12-14). The expression of miRNAs is remarkably deregulated in EOC, implying the involvement of miRNAs in the initiation and progression of this disease (12-14). In the current study, the data indicated that miR-298 expression in human EOC tissues was significantly downregulated compared with that in normal controls, and negatively correlated with the expression of EZH2 mRNA. miR-298 downregulation and EZH2 upregulation were identified to be significantly associated with high clinical stage and pathological grade of EOC patients. In addition, the ectopic expression of miR-298 could efficiently inhibit cell migration and invasion. Notably, the overexpression of EZH2 could restore the cell migration and invasion abilities suppressed by miR-298. These results linked EZH2 to the miRNA miR-298, and demonstrated their roles in regulating the malignant phenotypes of EOC cells and the aggressive cancer progression of EOC patients.

Aberrant expression of miR-298 has been observed in ovarian cancer tissues (15), serum from men with metastatic castration-resistant prostate cancer (21) and breast cancer cell lines (22). However, the function and mechanism of this miRNA regulation in human cancers remain unclear. Gui et al (15) previously reported that EZH2 expression could be concomitantly and significantly reduced by miR-298 overexpression in EOC cells. As a catalytic subunit of polycomb repressive complex 2, EZH2 exhibits intrinsic histone lysine methyltransferase activity on histone H3 Lys-9 and -27 or histone H1 Lys-26 (23). EZH2 acts as a recruitment platform for DNA methyltransferases, and is essential for DNA methylation of EZH2-target promoters (24). Notably, EZH2 also can silence gene expression by binding to the promoter of its target genes directly (25). Previous studies have reported the overexpression of EZH2 in several types of human cancers and its association with aggressiveness and poor prognosis in breast (26) and prostate cancer (27). EZH2 is often upregulated in EOC cells, and can promote cell proliferation, inhibit apoptosis and enhance angiogenesis in $\operatorname{EOC}(28,29)$. Thus, the present authors speculated that the dysregulation of miR-298 may contribute to EZH2-mediated malignant phenotypes in 
EOC cells. The present study confirmed the inverse correlation existing between miR-298 and EZH2 mRNA expression levels in EOC tissues based on a large cohort of clinical samples. Notably, statistical analysis revealed remarkable associations between miR-298 downregulation and/or EZH2 upregulation and aggressive clinical phenotypes of EOC patients. In essence, this provided the possibility that the dysregulation of the miR-298-EZH2 axis may lead to occurrence and progression in EOC.

Migration and invasion, resulting in high cell mortality, represent two major attributes of malignant tumors (30). In the current study, it was validated that miR-298 downregulation may result in the inhibition of the migration and invasion of EOC cells. To the best of our knowledge, there are no published data on the role of miR-298 regarding migration and invasion in cancer cells. Additionally, via functional studies on EZH2, it was noticed that the overexpression of EZH2 could restore the inhibitory effects of miR-298 on protein levels, cell migration and cell invasion abilities, further confirming that miR-298 exhibited an EZH2-dependent stimulatory activity in the regulation of migration and invasion in EOC cells.

In conclusion, our data offer convincing evidence that the dysregulation of the miR-298-EZH2 axis may be important in tumor progression of EOC patients. The present findings also confirmed a tumor-suppressive role of miR-298 in modulating EOC cell motility by regulating the expression of EZH2, implying its potential as a novel miRNA-based therapeutic target for the treatment of human EOC.

\section{References}

1. Vargas-Hernández VM, Moreno-Eutimio MA Acosta-Altamirano G and Vargas-Aguilar VM: Management of recurrent epithelial ovarian cancer. Gland Surg 3: 198-202, 2014

2. Xu L, Cai J, Yang Q, Ding H, Wu L, Li T and Wang Z: Prognostic significance of several biomarkers in epithelial ovarian cancer: A meta-analysis of published studies. J Cancer Res Clin Oncol 139: 1257-1277, 2013.

3. Yeh YM, Chuang CM, Chao KC and Wang LH: MicroRNA-138 suppresses ovarian cancer cell invasion and metastasis by targeting SOX4 and HIF-1 $\alpha$. Int J Cancer 133: 867-878, 2013

4. Huang L, Lv W and Zhao X: CD24 as a molecular marker in ovarian cancer: A literature review. Cancer Transl Med 2: 29-32, 2016.

5. Coleman RL, Monk BJ, Sood AK and Herzog TJ: Latest research and treatment of advanced-stage epithelial ovarian cancer. Nat Rev Clin Oncol 10: 211-224, 2013.

6. Minig L, Otaño L, Diaz-Padilla I, Alvarez Gallego R, Patrono MG and Valero de Bernabé J: Therapeutic management of epithelial ovarian cancer during pregnancy. Clin Transl Oncol 15: 259-264, 2013.

7. Hata A and Lieberman J: Dysregulation of microRNA biogenesis and gene silencing in cancer. Sci Signal 8: re3, 2015.

8. Shen J and Hung MC: Signaling-mediated regulation of MicroRNA processing. Cancer Res 75: 783-791, 2015.

9. Farazi TA, Hoell JI, Morozov P and Tuschl T: MicroRNAs in human cancer. Adv Exp Med Biol 774: 1-20, 2013.

10. Zaman MS, Maher DM, Khan S, Jaggi M and Chauhan SC: Current status and implications of microRNAs in ovarian cancer diagnosis and therapy. J Ovarian Res 5: 44, 2012.

11. Mansoori B, Mohammadi A, Shirjang S and Baradaran B: Micro-RNAs: The new potential biomarkers in cancer diagnosis, prognosis and cancer therapy. Cell Mol Biol (Noisy-le-grand) 61: $1-10,2015$.

12. Wang YQ, Guo RD, Guo RM, Sheng W and Yin LR: MicroRNA-182 promotes cell growth, invasion, and chemoresistance by targeting programmed cell death 4 (PDCD4) in human ovarian carcinomas. J Cell Biochem 114: 1464-1473, 2013.
13. Zhang H, Wang Q, Zhao Q and Di W: MiR-124 inhibits the migration and invasion of ovarian cancer cells by targeting SphK1. J Ovarian Res 6: 84, 2013.

14. Wu H, Xiao Z, Wang K, Liu W and Hao Q: MiR-145 is downregulated in human ovarian cancer and modulates cell growth and invasion by targeting p70S6K1 and MUC1. Biochem Biophys Res Commun 441: 693-700, 2013.

15. Gui T, Bai H, Zeng J, Zhong Z, Cao D, Cui Q, Chen J, Yang J and Shen K: Tumor heterogeneity in the recurrence of epithelial ovarian cancer demonstrated by polycomb group proteins. Onco Targets Ther 7: 1705-1716, 2014.

16. Pereira A, Pérez-Medina T, Magrina JF, Magtibay PM, Rodríguez-Tapia A, Peregrin I, Mendizabal E and OrtizQuintana L: International Federation of Gynecology and Obstetrics staging classification for cancer of the ovary, fallopian tube, and peritoneum: Estimation of survival in patients with node-positive epithelial ovarian cancer. Int J Gynecol Cancer 25: 49-54, 2015.

17. Livak KJ and Schmittgen TD: Analysis of relative gene expression data using real-time quantitative PCR and the 2(-Delta Delta C(T)) Method. Methods 25: 402-408, 2001.

18. Zhang Q, Padi SK, Tindall DJ and Guo B: Polycomb protein EZH2 suppresses apoptosis by silencing the proapoptotic miR-31. Cell Death Dis 5: e1486, 2014.

19. Semaan A, Qazi AM, Seward S, Chamala S, Bryant CS, Kumar S, Morris R, Steffes CP, Bouwman DL, Munkarah AR, et al: MicroRNA-101 inhibits growth of epithelial ovarian cancer by relieving chromatin-mediated transcriptional repression of p21(waf1/cip1). Pharm Res 28: 3079-3090, 2011.

20. Kim TH, Song JY, Park H, Jeong JY, Kwon AY, Heo JH, Kang H, Kim $\mathrm{G}$ and An HJ: miR-145, targeting high-mobility group A2, is a powerful predictor of patient outcome in ovarian carcinoma. Cancer Lett 356: 937-945, 2015.

21. Selth LA, Townley S, Gillis JL, Ochnik AM, Murti K, Macfarlane RJ, Chi KN, Marshall VR, Tilley WD and Butler LM: Discovery of circulating microRNAs associated with human prostate cancer using a mouse model of disease. Int J Cancer 131: 652-661, 2012.

22. Bao L, Hazari S, Mehra S, Kaushal D, Moroz K and Dash S: Increased expression of P-glycoprotein and doxorubicin chemoresistance of metastatic breast cancer is regulated by miR-298. Am J Pathol 180: 2490-2503, 2012.

23. Han Li C and Chen Y: Targeting EZH2 for cancer therapy: Progress and perspective. Curr Protein Pept Sci 16: 559-570, 2015.

24. Verma SK: Inhibition of the histone lysine methyltransferase EZH2 for the treatment of cancer. Curr Top Med Chem 15: 714-719, 2015.

25. Li LY: EZH2: Novel therapeutic target for human cancer. Biomedicine (Taipei) 4: 1, 2014.

26. Gong Y, Huo L, Liu P, Sneige N, Sun X, Ueno NT, Lucci A, Buchholz TA, Valero V and Cristofanilli M: Polycomb group protein EZH2 is frequently expressed in inflammatory breast cancer and is predictive of worse clinical outcome. Cancer 117: 5476-5484, 2011

27. Yang YA and $\mathrm{Yu}$ J: EZH2, an epigenetic driver of prostate cancer. Protein Cell 4: 331-341, 2013.

28. Li T, Cai J, Ding H, Xu L, Yang Q and Wang Z: EZH2 participates in malignant biological behavior of epithelial ovarian cancer through regulating the expression of BRCA1. Cancer Biol Ther 15: 271-278, 2014.

29. Liu L, Guo J, Yu L, Cai J, Gui T, Tang H, Song L, Wang J, Han F, Yang C, et al: miR-101 regulates expression of EZH2 and contributes to progression of and cisplatin resistance in epithelial ovarian cancer. Tumour Biol 35: 12619-12626, 2014.

30. Martin TA, Ye L, Sanders AJ, Lane J and Jiang WG: Cancer invasion and metastasis: Molecular and cellular perspective. In: Metastatic Cancer: Clinical and Biological Perspectives. Jandial R (ed). Madame Curie Bioscience Database. Landes Bioscience, Austin, TX, 2000-2013. 